\title{
Dengue Virus Infection and Associated Risk Factors in Africa: A Systematic Review and Meta-Analysis
}

\author{
Gaspary O. Mwanyika 1,2,3 ${ }^{\mathbb{D}}$, Leonard E. G. Mboera ${ }^{1} \mathbb{D}$, Sima Rugarabamu $1,2,4 \mathbb{D}^{1}$, Baraka Ngingo $^{1,5}$, \\ Calvin Sindato ${ }^{1,6}$, Julius J. Lutwama ${ }^{7}$, Janusz T. Paweska ${ }^{8}$ and Gerald Misinzo ${ }^{1,2, *(D)}$
}

1 SACIDS Africa Centre of Excellence for Infectious Diseases, Sokoine University of Agriculture, P.O. Box 3297 Morogoro, Tanzania; gaspary.mwanyika@sacids.org (G.O.M.); leonard.mboera@sacids.org (L.E.G.M.); sima.rugarabamu@sacids.org (S.R.); ngingobaraka@gmail.com (B.N.); calvin.sindato@sacids.org (C.S.)

2 Department of Veterinary Microbiology, Parasitology and Biotechnology, Sokoine University of Agriculture, P.O. Box 3015 Morogoro, Tanzania

3 Department of Health Science and Technology, Mbeya University of Science and Technology, P.O. Box 131 Mbeya, Tanzania

4 Department of Microbiology and Immunology, Muhimbili University of Health and Allied Sciences, P.O. Box 65595 Dar es Salaam, Tanzania

5 Biology Department, St. John's University of Tanzania, P.O. Box 47 Dodoma, Tanzania

6 Tabora Research Centre, National Institute for Medical Research, P.O. Box 482 Tabora, Tanzania

7 Department of Arbovirology, Emerging and Re-emerging Infectious Diseases, Uganda Virus Research Institute, P.O. Box 49 Entebbe, Uganda; jilutwama03@yahoo.com

8 National Health Laboratory Service, National Institute for Communicable Diseases, Sandringham, 2192 Johannesburg, South Africa; janusz@gmail.com

check for updates

Citation: Mwanyika, G.O.; Mboera, L.E.G.; Rugarabamu, S.; Ngingo, B.; Sindato, C.; Lutwama, J.J.; Paweska, J.T.; Misinzo, G. Dengue Virus Infection and Associated Risk Factors in Africa: A Systematic Review and Meta-Analysis. Viruses 2021, 13, 536. https://doi.org/10.3390/v13040536

Academic Editor: Luciana Barros de Arruda

Received: 25 February 2021

Accepted: 4 March 2021

Published: 24 March 2021

Publisher's Note: MDPI stays neutral with regard to jurisdictional claims in published maps and institutional affiliations.

Copyright: (c) 2021 by the authors. Licensee MDPI, Basel, Switzerland. This article is an open access article distributed under the terms and conditions of the Creative Commons Attribution (CC BY) license (https:/ / creativecommons.org/licenses/by/ $4.0 /)$.
* Correspondence: gerald.misinzo@sacids.org

\begin{abstract}
Dengue contributes a significant burden on global public health and economies. In Africa, the burden of dengue virus (DENV) infection is not well described. This review was undertaken to determine the prevalence of dengue and associated risk factors. A literature search was done on PubMed/MEDLINE, Scopus, Embase, and Google Scholar databases to identify articles published between 1960 and 2020. Meta-analysis was performed using a random-effect model at a 95\% confidence interval, followed by subgroup meta-analysis to determine the overall prevalence. Between 1960 and 2020, 45 outbreaks were identified, of which 17 and 16 occurred in East and West Africa, respectively. Dengue virus serotype 1 (DENV-1) and DENV-2 were the dominant serotypes contributing to $60 \%$ of the epidemics. Of 2211 cases reported between 2009 and 2020; 1954 (88.4\%) were reported during outbreaks. Overall, the prevalence of dengue was 29\% (95\% CI: $20-39 \%$ ) and 3\% (95\% CI: $1-5 \%$ ) during the outbreak and non-outbreak periods, respectively. Old age (6/21 studies), lack of mosquito control (6/21), urban residence (4/21), climate change (3/21), and recent history of travel $(3 / 21)$ were the leading risk factors. This review reports a high burden of dengue and increased risk of severe disease in Africa. Our findings provide useful information for clinical practice and health policy decisions to implement effective interventions.
\end{abstract}

Keywords: dengue; prevalence; risk factors; Africa

\section{Introduction}

Dengue is an important arboviral disease, with the highest incidence in tropical and subtropical regions, with a potential to spread into other geographical areas. In the past four decades, dengue has caused a significant impact on human health and national economies [1,2]. Approximately 390 million people are infected with dengue virus (DENV) annually. Of these, 96 million develop clinical manifestations that lead to 500,000 hospitalizations and 25,000 deaths, annually [3]. Dengue is caused by an RNA virus of the family Flaviviridae that is transmitted to humans through a bite of infected Aedes mosquitoes. 
Dengue virus exists in four genetically related but antigenically distinct serotypes (DENV1-4), each with the ability to cause self-limiting fevers to fatal conditions such as dengue hemorrhagic fever (DHF) and dengue shock syndrome (DSS). Although dengue infection confers lifelong immunity after primary infection by one serotype, secondary infection by heterologous serotypes or virulent strains increases the risk of severe disease [4]. During the 19th century, dengue epidemics in Africa were initially reported in the Zanzibar Islands in 1823 and 1870, Burkina Faso in 1925, and South Africa between 1926 and 1927 [5]. In the 1960s, laboratory-confirmed outbreaks started being reported in many other African countries [5]. All four serotypes (DENV-1-4) have been reported in the continent, with DENV-1 and DENV-2 being reported most frequently [6,7]. Despite increasing reports of dengue in Africa, its burden in different epidemiological contexts is not well described. This could be due to inadequate laboratory capacity to differentiate dengue from other febrile illnesses, such as malaria, chikungunya, Zika, yellow fever, typhoid fever, and leptospirosis, that share a similar clinical presentation and geographical distribution [8-10]. The objective of this systematic review and meta-analysis was to analyze the prevalence of dengue infection and associated risk factors in Africa.

\section{Materials and Methods}

\subsection{Search Strategy and Selection Criteria}

This review was performed according to Preferred Reporting Items for Systematic Reviews and Meta-analyses (PRISMA) guidelines [11], and the protocol registered in PROSPERO (CDR420202105579). PubMed/MEDLINE, Scopus, Embase, and Google Scholar databases were searched for articles for a period of three months from October to December 2020. Additional literature was searched from African Journals Online, World Health Organization, and Program for Monitoring Emerging Diseases (ProMED) databases. The key search terms were: (Dengue) AND (Africa = list of countries) AND ("Outbreak" OR "Prevalence" OR "Co-morbidities" OR "Risk factors") (Appendix A). The primary studies describing outbreak incidence, dengue prevalence based on ribonucleic acid (RNA) and non-structural protein 1 (NS1) antigen, co-morbidities, and potential risk factors in the African continent published between 1960 and 2020 were considered for review. We excluded studies with abstracts only, dengue cases in studies not involving human subjects, articles in languages other than English, review papers, and studies with incomplete data. Dengue infection was defined as febrile illness presenting with fever and at least two of the following clinical manifestations: headache, retro-orbital pain, myalgia, arthralgia, rash, hemorrhagic manifestations, and leukopenia confirmed by laboratory criteria through the detection of DENV RNA using reverse transcription polymerase chain reaction (RT-PCR) or NS1 antigens using enzyme-linked immunosorbent assay (ELISA) and/or rapid tests.

\subsection{Data Extraction and Management}

Records on the authors, geographical origin, setting (hospital versus community), study design, number of dengue cases, total participants tested and epidemiological context (outbreak versus non-outbreak), detected DENV serotypes, co-morbidities, and potential risk factors of dengue infection were extracted into a Microsoft Excel spreadsheet (Excel 2019, Microsoft Corp., Redmond, WA, USA). The duplicates were removed using Rayyan web application software for systematic review [12].

\subsection{Quality Assessment}

The methodological quality of selected prevalence studies was evaluated by two reviewers using a quality assessment checklist adapted from Hoy and others [13]. Risk of bias was assessed using nine domains: target population, sampling frame, sample selection method, likelihood of non-response bias, data source, case definition, study instrument that measured the parameter of interest, mode of data collection, and numerator and denominator of the parameter of interest. The risk of bias levels was low $($ score $=0$ ) or high (score $=1$ ), and the overall risk of bias was defined as low (score $0-3)$, moderate 
(4-6), and high (7-9) (Table S1). Any discrepancy was resolved through discussion with a third reviewer.

\subsection{Statistical Analysis}

The extracted data were pooled using MetaXL version 5.3 software (EpiGear International Pty Ltd., Queensland, QLD, Australia). A random effect model was used to estimate the overall prevalence and 95\% confidence intervals (CI), and results were presented in forest plots. The percentage of heterogeneity between studies was quantified using $\mathrm{I}^{2}$ and chi-square tests, and $\mathrm{I}^{2} \geq 50 \%$ was considered significant. Sensitivity analysis to test the effect of each study on summary prevalence, by excluding each study step by step, was used to evaluate the robustness of overall prevalence. A funnel plot and Egger's regression test were used to detect publication bias. All results with $p$-values $<0.05$ were considered statistically significant. Descriptive statistics, narrative synthesis, and relevant figures were used to summarize the information where statistical pooling was not possible.

\section{Results}

\subsection{Search Results and Characteristics of Selected Studies}

A total of 2170 records were retrieved from database searches. After duplicates removal and screening, 43 studies were finally included in the review (Figure 1). The methodological quality of studies ranged from low (0-3 score, 37 studies) to moderate (4-6 score, 6 studies). No study had a high risk of bias, six (13.9\%) studies had moderate risk, and $37(86.1 \%)$ had low risk (Table S2). Out of 43 studies, 34 were prospective crosssectional, six were retrospective cross-sectional, two were prospective cohort, and one was a case-control study (Table 1).

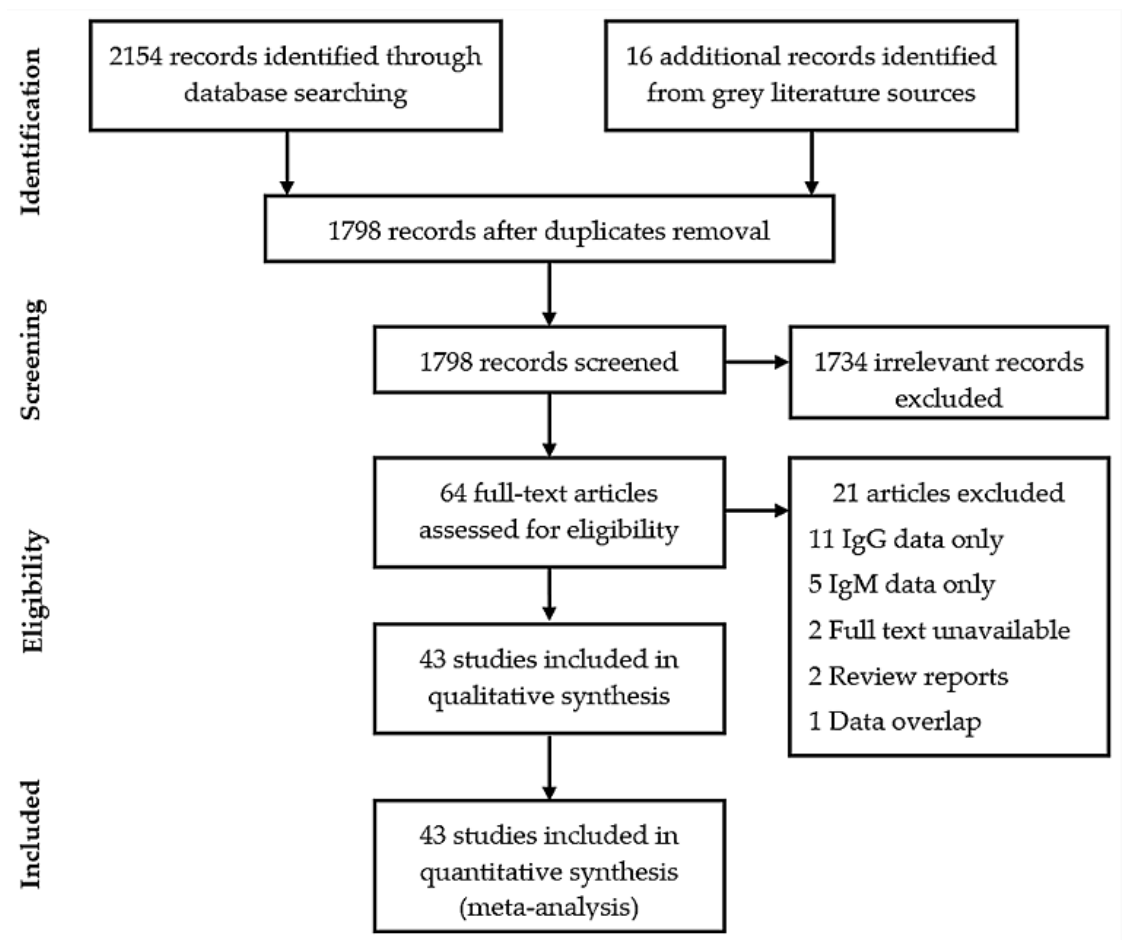

Figure 1. PRISMA flow chart illustrating literature selection and inclusion process. 
Table 1. The characteristics of studies included in the systematic review and meta-analysis by country, region, design, and population.

\begin{tabular}{|c|c|c|c|c|c|c|c|c|c|}
\hline $\mathbf{s} / \mathbf{n}$ & Reference & Country & Region & Design & Population & Cases & $\begin{array}{l}\text { Sample } \\
\text { Size }\end{array}$ & $\begin{array}{c}\text { Epidemiological } \\
\text { Context }\end{array}$ & $\begin{array}{c}\text { Risk of } \\
\text { Bias }\end{array}$ \\
\hline 1 & $\begin{array}{l}\text { Ratsitorahina } \\
\text { et al. [14] }\end{array}$ & Madagascar & East Africa & $\begin{array}{l}\text { Retrospective } \\
\text { cross-sectional }\end{array}$ & $\begin{array}{l}\text { Dengue } \\
\text { confirmed febrile }\end{array}$ & 24 & 55 & Outbreak & Low \\
\hline 2 & $\begin{array}{l}\text { Leroy et al. } \\
\text { [15] }\end{array}$ & Gabon & $\begin{array}{l}\text { Central } \\
\text { Africa }\end{array}$ & $\begin{array}{l}\text { Prospective } \\
\text { cross-sectional }\end{array}$ & $\begin{array}{l}\text { Dengue } \\
\text { confirmed febrile }\end{array}$ & 54 & 773 & Outbreak & Low \\
\hline 3 & $\begin{array}{l}\text { Nkoghe et al. } \\
\text { [16] }\end{array}$ & Gabon & $\begin{array}{l}\text { Central } \\
\text { Africa }\end{array}$ & $\begin{array}{l}\text { Prospective } \\
\text { cross-sectional }\end{array}$ & $\begin{array}{l}\text { Dengue } \\
\text { confirmed febrile }\end{array}$ & 53 & 433 & Outbreak & Low \\
\hline 4 & $\begin{array}{l}\text { Malik et al. } \\
\text { [17] }\end{array}$ & $\begin{array}{l}\text { Republic of } \\
\text { Sudan }\end{array}$ & $\begin{array}{l}\text { North } \\
\text { Africa }\end{array}$ & $\begin{array}{l}\text { Prospective } \\
\text { cross-sectional }\end{array}$ & $\begin{array}{l}\text { Dengue } \\
\text { confirmed febrile }\end{array}$ & 9 & 23 & Outbreak & Low \\
\hline 5 & $\begin{array}{l}\text { Phoutrides } \\
\text { et al. [18] }\end{array}$ & Mali & West Africa & $\begin{array}{l}\text { Retrospective } \\
\text { cross-sectional }\end{array}$ & Febrile patients & 0 & 95 & Non-outbreak & Moderate \\
\hline 6 & $\begin{array}{l}\text { Caron et al. } \\
\text { [19] }\end{array}$ & Gabon & $\begin{array}{l}\text { Central } \\
\text { Africa }\end{array}$ & $\begin{array}{l}\text { Prospective } \\
\text { cross-sectional }\end{array}$ & $\begin{array}{l}\text { Dengue } \\
\text { confirmed febrile }\end{array}$ & 376 & 4287 & Outbreak & Low \\
\hline 7 & $\begin{array}{l}\text { Hertz et al. } \\
\text { [20] }\end{array}$ & Tanzania & East Africa & $\begin{array}{l}\text { Prospective } \\
\text { cross-sectional }\end{array}$ & Febrile patients & 0 & 700 & Non-outbreak & Low \\
\hline 8 & $\begin{array}{l}\text { Vairo et al. } \\
\text { [21] }\end{array}$ & Tanzania & East Africa & $\begin{array}{l}\text { Prospective } \\
\text { cross-sectional }\end{array}$ & Febrile patients & 0 & 165 & Non-outbreak & Low \\
\hline 9 & $\begin{array}{l}\text { Faye et al. } \\
\text { [22] }\end{array}$ & Senegal & West Africa & $\begin{array}{l}\text { Prospective } \\
\text { cross-sectional }\end{array}$ & $\begin{array}{l}\text { Dengue } \\
\text { confirmed febrile }\end{array}$ & 196 & 696 & Outbreak & Low \\
\hline 10 & $\begin{array}{l}\text { Parreira et al. } \\
\text { [23] }\end{array}$ & Angola & $\begin{array}{l}\text { South } \\
\text { Africa }\end{array}$ & $\begin{array}{l}\text { Prospective } \\
\text { cross-sectional }\end{array}$ & $\begin{array}{l}\text { Dengue } \\
\text { confirmed febrile }\end{array}$ & 25 & 29 & Outbreak & Low \\
\hline 11 & $\begin{array}{l}\text { Chipwaza } \\
\text { et al. [24] }\end{array}$ & Tanzania & East Africa & $\begin{array}{l}\text { Prospective } \\
\text { cross-sectional }\end{array}$ & $\begin{array}{l}\text { Dengue } \\
\text { confirmed febrile }\end{array}$ & 29 & 364 & Non-outbreak & Low \\
\hline 12 & $\begin{array}{l}\text { Elduma et al. } \\
\text { [25] }\end{array}$ & $\begin{array}{l}\text { Republic of } \\
\text { Sudan }\end{array}$ & $\begin{array}{l}\text { North } \\
\text { Africa }\end{array}$ & $\begin{array}{l}\text { Prospective } \\
\text { cross-sectional }\end{array}$ & $\begin{array}{l}\text { Dengue } \\
\text { confirmed febrile }\end{array}$ & 1 & 39 & Non-outbreak & Moderate \\
\hline 13 & $\begin{array}{l}\text { L'Azou et al. } \\
\text { [26] }\end{array}$ & $\begin{array}{l}\text { Côte } \\
\text { d'Ivoire }\end{array}$ & West Africa & $\begin{array}{l}\text { Prospective } \\
\text { cross-sectional }\end{array}$ & Febrile patients & 1 & 796 & Non-outbreak & Low \\
\hline 14 & $\begin{array}{l}\text { Ellis et al. } \\
\text { [27] }\end{array}$ & Kenya & East Africa & $\begin{array}{l}\text { Prospective } \\
\text { cross-sectional }\end{array}$ & $\begin{array}{l}\text { Dengue } \\
\text { confirmed febrile }\end{array}$ & 155 & 267 & Outbreak & Low \\
\hline 15 & $\begin{array}{l}\text { Konongoi } \\
\text { et al. [28] }\end{array}$ & Kenya & East Africa & $\begin{array}{l}\text { Prospective } \\
\text { cross-sectional }\end{array}$ & $\begin{array}{l}\text { Dengue } \\
\text { confirmed febrile }\end{array}$ & 29 & 364 & Non-outbreak & Low \\
\hline 16 & $\begin{array}{l}\text { Ngoi et al. } \\
\text { [29] }\end{array}$ & Kenya & East Africa & $\begin{array}{l}\text { Prospective } \\
\text { cohort }\end{array}$ & $\begin{array}{l}\text { Dengue } \\
\text { confirmed febrile }\end{array}$ & 43 & 489 & Non-outbreak & Moderate \\
\hline 17 & $\begin{array}{l}\text { Gonidec et al. } \\
\text { [30] }\end{array}$ & Djibouti & East Africa & $\begin{array}{l}\text { Retrospective } \\
\text { cross-sectional }\end{array}$ & $\begin{array}{l}\text { Dengue } \\
\text { confirmed febrile }\end{array}$ & 78 & 354 & Outbreak & Low \\
\hline 18 & $\begin{array}{l}\text { Vairo et al. } \\
\text { [31] }\end{array}$ & Tanzania & East Africa & $\begin{array}{l}\text { Prospective } \\
\text { cross-sectional }\end{array}$ & $\begin{array}{l}\text { Dengue } \\
\text { confirmed febrile }\end{array}$ & 101 & 483 & Outbreak & Low \\
\hline 19 & $\begin{array}{l}\text { Hansperger } \\
\text { et al. [32] }\end{array}$ & Angola & $\begin{array}{l}\text { South } \\
\text { Africa }\end{array}$ & $\begin{array}{l}\text { Prospective } \\
\text { cross-sectional }\end{array}$ & $\begin{array}{l}\text { Dengue } \\
\text { confirmed febrile }\end{array}$ & 29 & 46 & Outbreak & Moderate \\
\hline 20 & $\begin{array}{l}\text { Abreu et al. } \\
\text { [33] }\end{array}$ & Angola & $\begin{array}{l}\text { South } \\
\text { Africa }\end{array}$ & $\begin{array}{l}\text { Retrospective } \\
\text { cross-sectional }\end{array}$ & $\begin{array}{l}\text { Dengue } \\
\text { confirmed febrile }\end{array}$ & 11 & 17 & Outbreak & Moderate \\
\hline 21 & Vu et al. [34] & Kenya & East Africa & $\begin{array}{l}\text { Prospective } \\
\text { cross-sectional }\end{array}$ & $\begin{array}{l}\text { Dengue } \\
\text { confirmed febrile }\end{array}$ & 82 & 1104 & Non-outbreak & Moderate \\
\hline 22 & $\begin{array}{l}\text { Oludele et al. } \\
\text { [35] }\end{array}$ & Mozambique & $\begin{array}{l}\text { South } \\
\text { Africa }\end{array}$ & $\begin{array}{l}\text { Prospective } \\
\text { cross-sectional }\end{array}$ & $\begin{array}{l}\text { Dengue } \\
\text { confirmed febrile }\end{array}$ & 60 & 192 & Outbreak & Low \\
\hline 23 & $\begin{array}{l}\text { Simo et al. } \\
\text { [36] }\end{array}$ & Cameroon & West Africa & $\begin{array}{l}\text { Prospective } \\
\text { cross-sectional }\end{array}$ & $\begin{array}{l}\text { Dengue } \\
\text { confirmed febrile }\end{array}$ & 10 & 91 & Outbreak & Low \\
\hline 24 & $\begin{array}{l}\text { Hercik et al. } \\
\text { [37] }\end{array}$ & Tanzania & East Africa & $\begin{array}{l}\text { Prospective } \\
\text { cross-sectional }\end{array}$ & $\begin{array}{l}\text { Dengue } \\
\text { confirmed febrile }\end{array}$ & 1 & 191 & Non-outbreak & Low \\
\hline 25 & $\begin{array}{l}\text { Obonyo et al. } \\
\text { [38] }\end{array}$ & Kenya & East Africa & $\begin{array}{l}\text { Prospective } \\
\text { cross-sectional }\end{array}$ & $\begin{array}{l}\text { Dengue } \\
\text { confirmed febrile }\end{array}$ & 30 & 381 & Outbreak & Low \\
\hline 26 & $\begin{array}{l}\text { Makiala et al. } \\
\text { [39] }\end{array}$ & $\mathrm{DRC}^{*}$ & $\begin{array}{l}\text { Central } \\
\text { Africa }\end{array}$ & $\begin{array}{l}\text { Prospective } \\
\text { cross-sectional }\end{array}$ & $\begin{array}{l}\text { Dengue } \\
\text { confirmed febrile }\end{array}$ & 16 & 453 & Non-outbreak & Low \\
\hline 27 & $\begin{array}{l}\text { Amoako et al. } \\
\text { [40] }\end{array}$ & Ghana & West Africa & $\begin{array}{l}\text { Retrospective } \\
\text { cross-sectional }\end{array}$ & $\begin{array}{l}\text { Dengue } \\
\text { confirmed febrile }\end{array}$ & 2 & 166 & Non-outbreak & Low \\
\hline 28 & $\begin{array}{l}\text { Humphrey } \\
\text { et al. [41] }\end{array}$ & Ghana & West Africa & $\begin{array}{l}\text { Retrospective } \\
\text { cross-sectional }\end{array}$ & $\begin{array}{l}\text { Dengue } \\
\text { confirmed febrile }\end{array}$ & 4 & 150 & Non-outbreak & Low \\
\hline 29 & $\begin{array}{l}\text { Tarnagda } \\
\text { et al. [42] }\end{array}$ & $\begin{array}{l}\text { Burkina } \\
\text { Faso }\end{array}$ & West Africa & $\begin{array}{l}\text { Prospective } \\
\text { cross-sectional }\end{array}$ & $\begin{array}{l}\text { Dengue } \\
\text { confirmed febrile }\end{array}$ & 19 & 35 & Outbreak & Low \\
\hline 30 & $\begin{array}{l}\text { Yousseu et al. } \\
\text { [43] }\end{array}$ & Cameroon & West Africa & $\begin{array}{l}\text { Prospective } \\
\text { cross-sectional }\end{array}$ & $\begin{array}{l}\text { Dengue } \\
\text { confirmed febrile }\end{array}$ & 8 & 114 & Non-outbreak & Low \\
\hline 31 & $\begin{array}{l}\text { Hamid et al. } \\
{[44]}\end{array}$ & $\begin{array}{l}\text { Republic of } \\
\text { Sudan }\end{array}$ & $\begin{array}{l}\text { North } \\
\text { Africa }\end{array}$ & $\begin{array}{l}\text { Prospective } \\
\text { cross-sectional }\end{array}$ & $\begin{array}{l}\text { Dengue } \\
\text { confirmed febrile }\end{array}$ & 4 & 106 & Non-outbreak & Low \\
\hline 32 & $\begin{array}{l}\text { Degife et al. } \\
\text { [45] }\end{array}$ & Ethiopia & East Africa & case control & Dengue suspects & 42 & 69 & Outbreak & Low \\
\hline 33 & $\begin{array}{l}\text { Ghweil et al. } \\
\text { [46] }\end{array}$ & Egypt & $\begin{array}{l}\text { North } \\
\text { Africa }\end{array}$ & $\begin{array}{l}\text { Prospective } \\
\text { cohort }\end{array}$ & $\begin{array}{l}\text { Dengue } \\
\text { confirmed febrile }\end{array}$ & 100 & 200 & Outbreak & Low \\
\hline
\end{tabular}


Table 1. Cont.

\begin{tabular}{|c|c|c|c|c|c|c|c|c|c|}
\hline $\mathbf{s} / \mathbf{n}$ & Reference & Country & Region & Design & Population & Cases & $\begin{array}{c}\text { Sample } \\
\text { Size }\end{array}$ & $\begin{array}{c}\text { Epidemiological } \\
\text { Context }\end{array}$ & $\begin{array}{c}\text { Risk of } \\
\text { Bias }\end{array}$ \\
\hline 34 & $\begin{array}{l}\text { Ahmed et al. } \\
\text { [47] }\end{array}$ & $\begin{array}{l}\text { Republic of } \\
\text { Sudan }\end{array}$ & $\begin{array}{l}\text { North } \\
\text { Africa }\end{array}$ & $\begin{array}{l}\text { Prospective } \\
\text { cross-sectional }\end{array}$ & $\begin{array}{l}\text { Dengue } \\
\text { confirmed febrile }\end{array}$ & 32 & 204 & Outbreak & Low \\
\hline 35 & $\begin{array}{l}\text { Proesmans } \\
\text { et al. [48] }\end{array}$ & $\mathrm{DRC}^{*}$ & $\begin{array}{l}\text { Central } \\
\text { Africa }\end{array}$ & $\begin{array}{l}\text { Prospective } \\
\text { cross-sectional }\end{array}$ & $\begin{array}{l}\text { Dengue } \\
\text { confirmed febrile }\end{array}$ & 14 & 235 & Non-outbreak & Low \\
\hline 36 & $\begin{array}{l}\text { Ayolabi et al. } \\
\text { [49] }\end{array}$ & Nigeria & West Africa & $\begin{array}{l}\text { Prospective } \\
\text { cross-sectional }\end{array}$ & $\begin{array}{l}\text { Dengue } \\
\text { confirmed febrile }\end{array}$ & 11 & 130 & Non-outbreak & Low \\
\hline 37 & $\begin{array}{l}\text { Boyce et al. } \\
{[50]}\end{array}$ & Uganda & East Africa & $\begin{array}{l}\text { Prospective } \\
\text { cross-sectional }\end{array}$ & Febrile patients & 0 & 1416 & Non-outbreak & Low \\
\hline 38 & $\begin{array}{l}\text { Chipwaza } \\
\text { et al. [51] }\end{array}$ & Tanzania & East Africa & $\begin{array}{l}\text { Prospective } \\
\text { cross-sectional }\end{array}$ & $\begin{array}{l}\text { Dengue } \\
\text { confirmed febrile }\end{array}$ & 29 & 294 & Outbreak & Low \\
\hline 39 & $\begin{array}{l}\text { Dieng et al. } \\
\text { [52] }\end{array}$ & Senegal & West Africa & $\begin{array}{l}\text { Prospective } \\
\text { cross-sectional }\end{array}$ & $\begin{array}{l}\text { Dengue } \\
\text { confirmed febrile }\end{array}$ & 3 & 104 & Non-outbreak & Low \\
\hline 40 & Im et al. [53] & $\begin{array}{l}\text { Burkina } \\
\text { Faso }\end{array}$ & West Africa & $\begin{array}{l}\text { Prospective } \\
\text { cross-sectional }\end{array}$ & $\begin{array}{l}\text { Dengue } \\
\text { confirmed febrile }\end{array}$ & 141 & 241 & Outbreak & Low \\
\hline 41 & $\begin{array}{l}\text { Eldigail et al. } \\
\text { [54] }\end{array}$ & $\begin{array}{l}\text { Republic of } \\
\text { Sudan }\end{array}$ & $\begin{array}{l}\text { North } \\
\text { Africa }\end{array}$ & $\begin{array}{l}\text { Prospective } \\
\text { cross-sectional }\end{array}$ & $\begin{array}{l}\text { Dengue } \\
\text { confirmed febrile }\end{array}$ & 23 & 100 & Outbreak & Low \\
\hline 42 & $\begin{array}{l}\text { Masika et al. } \\
\text { [55] }\end{array}$ & Kenya & East Africa & $\begin{array}{l}\text { Prospective } \\
\text { cross-sectional }\end{array}$ & $\begin{array}{l}\text { Dengue } \\
\text { confirmed febrile }\end{array}$ & 5 & 560 & Non-outbreak & Low \\
\hline 43 & $\begin{array}{l}\text { Shah et al. } \\
\text { [56] }\end{array}$ & Kenya & East Africa & $\begin{array}{l}\text { Prospective } \\
\text { cross-sectional }\end{array}$ & $\begin{array}{l}\text { Dengue } \\
\text { confirmed febrile }\end{array}$ & 361 & 862 & Non-outbreak & Low \\
\hline
\end{tabular}

${ }^{*}$ DRC $=$ Democratic Republic of Congo.

\subsection{Dengue Virus Outbreaks and Serotype Distribution}

Since 1964, 45 dengue outbreaks were reported in 14 countries (Table 2). Most of the outbreaks occurred in East (17/45) and West (16/45) Africa. DENV-1 and DENV-2 were dominant serotypes in most of the outbreaks (Figure 2). During the past decade (2010-2020), there was an expansion of multiple DENV serotypes occurrence in Africa (Figure 3).

Table 2. Dengue virus outbreaks in Africa by year, country, sub-region, and serotype, 1964-2020.

\begin{tabular}{|c|c|c|c|c|c|}
\hline $\mathrm{S} / \mathrm{n}$ & Year of Outbreak & Country & Sub-Region & Serotype & Reference \\
\hline 1 & 1964 & Nigeria & West Africa & DENV-1/2 & [57] \\
\hline 2 & 1977 & Seychelles & East Africa & DENV-2 & [58] \\
\hline 3 & 1985 & Senegal & West Africa & DENV-2/4 & [59] \\
\hline 4 & 1985 & Somalia & East Africa & DENV-2 & [60] \\
\hline 5 & 2005 & Republic of Sudan & North Africa & DENV-3 & [17] \\
\hline 6 & 2007 & Gabon & Central Africa & DENV-2 & [15] \\
\hline 7 & 2008 & Mali & West Africa & DENV-3 & {$[61]^{* *}$} \\
\hline 8 & 2008 & Madagascar & East Africa & DENV-1 & [14] \\
\hline 9 & 2009 & Cape Verde & West Africa & $\mathrm{NR} *$ & {$[62]^{* * *}$} \\
\hline 10 & 2009 & Mauritius & East Africa & DENV-2 & [63] \\
\hline 11 & 2009 & Senegal & West Africa & DENV-3 & [22] \\
\hline 12 & 2010 & Gabon & Central Africa & NR & [61] \\
\hline 13 & 2010 & Côte d'Ivoire & West Africa & NR & [61] \\
\hline 14 & 2011 & Republic of Sudan & North Africa & DENV-3 & [17] \\
\hline 15 & 2012 & Republic of Sudan & North Africa & DENV-3 & [64] \\
\hline 16 & 2013 & Angola & Southern Africa & NR & [61] \\
\hline 17 & 2013 & Ethiopia & East Africa & DENV-2 & [65] \\
\hline 18 & 2013 & Kenya & East Africa & DENV-1/2 & [27] \\
\hline 19 & 2014 & Tanzania & East Africa & DENV-2 & [31] \\
\hline 20 & 2015 & Egypt & North Africa & DENV-1 & [62] \\
\hline 21 & 2015 & Ethiopia & East Africa & NR & [45] \\
\hline 22 & 2015 & Republic of Sudan & North Africa & DENV-1/3 & [47] \\
\hline 23 & 2016 & Burkina Faso & West Africa & DENV-1/3 & [53] \\
\hline
\end{tabular}


Table 2. Cont.

\begin{tabular}{cccccc}
\hline S/n & Year of Outbreak & Country & Sub-Region & Serotype & Reference \\
\hline 24 & 2016 & Reunion Islands & East Africa & DENV-1-4 & NR \\
25 & 2016 & Seychelles & East Africa & D6] & {$[61]$} \\
26 & 2016 & Burkina Faso & West Africa & DENV-2/3 & {$[42]$} \\
27 & 2016 & Angola & Southern Africa & DENV-3/4 & {$[23]$} \\
28 & 2016 & Djibouti & East Africa & DENV-1-3 & {$[30]$} \\
29 & 2016 & Mozambique & Southern Africa & DENV-2 & {$[35]$} \\
30 & 2017 & Burkina Faso & West Africa & DENV-1-3 & {$[62]$} \\
31 & 2017 & Côte d'Ivoire & West Africa & DENV-1-3 & {$[62]$} \\
32 & 2017 & Kenya & East Africa & NR & {$[62]$} \\
33 & 2017 & Côte d'Ivoire & West Africa & NR & {$[61]$} \\
34 & 2017 & Burkina Faso & West Africa & NR & {$[61]$} \\
35 & 2017 & Senegal & West Africa & NR & {$[61]$} \\
36 & 2017 & Reunion Islands & East Africa & DENV-1/2/4 & {$[66]$} \\
37 & 2018 & Tanzania & East Africa & DENV-1-4 & {$[51]$} \\
39 & 2018 & Senegal & West Africa & NR & {$[61]$} \\
40 & 2018 & Mauritania & North Africa & NR & {$[61]$} \\
41 & 2018 & Reunion Islands & East Africa & DENV-2 & {$[66]$} \\
42 & 2019 & Reunion Islands & East Africa & NR & {$[61]$} \\
44 & 2019 & Côte d'Ivoire & West Africa & DENV-1/3 & {$[61]$} \\
45 & 2019 & Tanzania & East Africa & DENV-1 & DENV-3
\end{tabular}

${ }^{*} \mathrm{NR}=$ serotype not reported; ${ }^{* *}$ ProMED-mail source: https://promedmail.org/ (accessed on 3 December 2020); ${ }^{* * *}$ WHO source: https://www.who.int/csr/don/en/ (accessed on 3 December 2020).

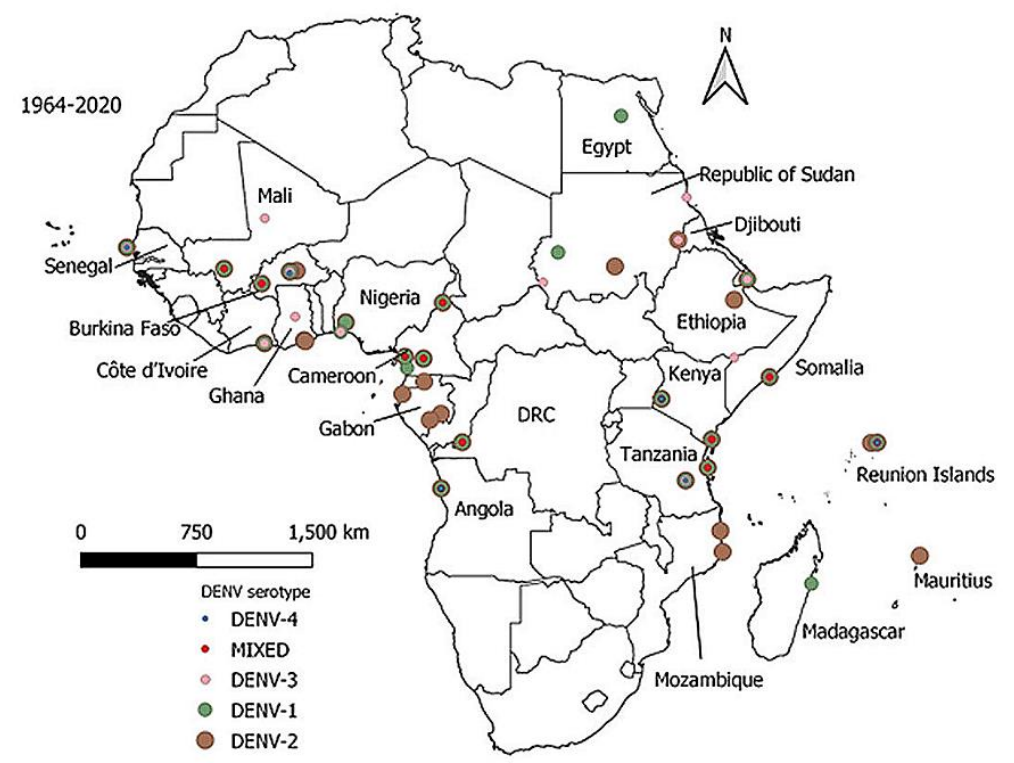

Figure 2. Distribution of dengue virus serotypes in Africa, 1964-2020. DENV-2 was the dominant serotype (brown), followed by DENV-1 (dark green), DENV-3 (light pink), Mixed serotypes (red) and DENV-4 (blue). The map was developed using Quantitative Geographic Information System (QGIS) open-source software version 3.16 available at https:/ / www.qgis.org/en/site/forusers/download (accessed on 12 February 2021). 


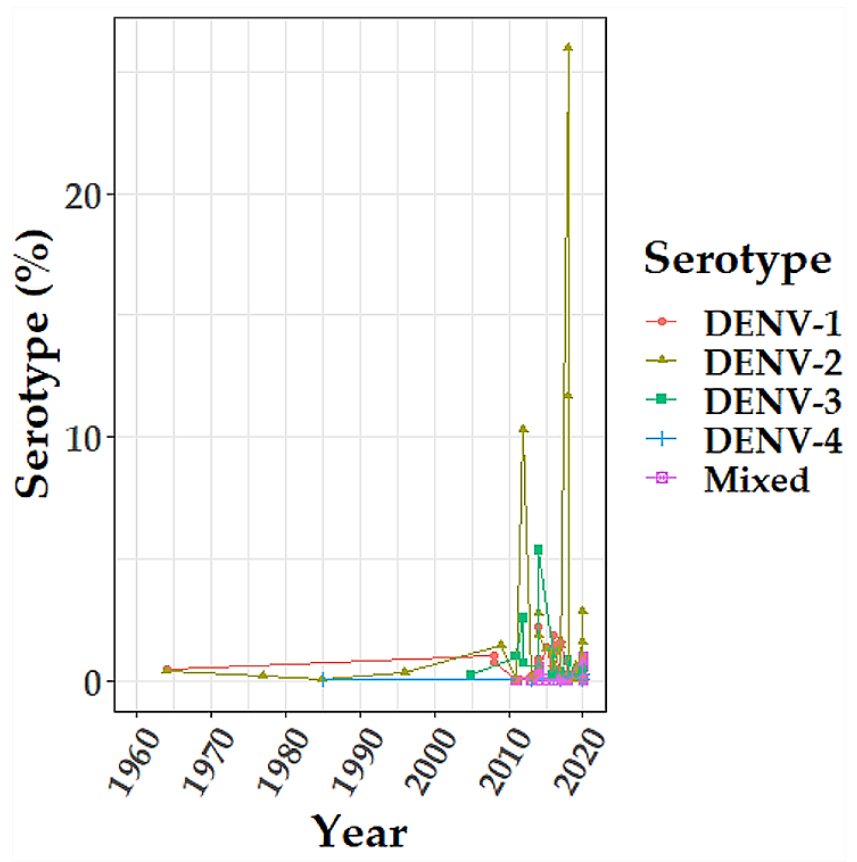

Figure 3. The longitudinal trend of dengue virus serotype proportion in Africa, 1964- 2020. The color codes represent DENV-1 (red), DENV-2 (dark khaki), DENV-3 (green), DENV-4 (blue), and Mixed serotype (pink). The graph was created using R software version 3.5.2 with a primary package ggplot2 at https:/ / cran.r-project.org/bin/windows/base/old/3.5.2/ (accessed on 3 December 2020).

\subsection{Dengue Prevalence}

Overall, the prevalence of DENV in Africa was 14\% (95\% CI, 9-19\%), N = 15,807). Substantial heterogeneity was found between studies during outbreak $\left(\mathrm{I}^{2}=99 \%, p<0.01\right)$ and non-outbreak $\left(\mathrm{I}^{2}=95 \%, p<0.01\right)$ periods (Figures 4 and 5$)$. Subgroup meta-analysis showed that the prevalence of DENV was $29 \%(95 \% \mathrm{CI}, 20-39 \%, \mathrm{~N}=8966)$ and 3\% (95\% CI, $1-5 \%, N=6841$ ) during outbreak and non-outbreak periods, respectively (Figure 6). Sensitivity analysis based on prospective cross-sectional studies $(n=34 / 43)$ showed that 33/34 studies had good precision on overall DENV prevalence (14\% (95\% CI, 9-20\%). One study had relatively low precision (12\% (95\% CI, 8-18\%) [23] (Table 3). Funnel plot asymmetry (Figure S1) and Egger's regression test $(p=0.0022)$ indicated an evidence of publication bias in the outbreak studies. No evidence of publication bias (Figure S2) was detected in the non-outbreak studies (Egger's regression test, $p=0.2633$ ).

Table 3. Sensitivity analysis of dengue virus prevalence based on prospective cross-sectional studies.

\begin{tabular}{cccc}
\hline Excluded Study & Pooled Prevalence (95\% CI) & $\left.\mathbf{I}^{\mathbf{2}} \mathbf{( 9 5 \%} \mathbf{C I}\right)$ & $p$-Value \\
\hline Outbreak Studies $(n=18)$ & & & \\
\hline Leroy et al., 2009 & $0.14(0.09,0.20)$ & $98.89(98.78,98.74)$ & $<0.01$ \\
Nkoghe et al., 2010 & $0.14(0.09,0.20)$ & $98.89(98.74,99.03)$ & $<0.01$ \\
Malik et al., 2011 & $0.13(0.08,019)$ & $98.89(98.73,99.02)$ & $<0.01$ \\
Caron et al., 2012 & $0.14(0.08,0.21)$ & $98.89(98.74,99.03)$ & $<0.01$ \\
Faye et al., 2014 & $0.13(0.08,0.19)$ & $98.89(98.64,98.96)$ & $<0.01$ \\
Parreira et al., 2014 & $0.12(0.08,0.18)$ & $98.89(98.74,99.03)$ & $<0.01$ \\
Ellis et al., 2015 & $0.13(0.08,0.18)$ & $98.89(98.54,98.90)$ & $<0.01$ \\
Vairo et al., 2016 & $0.13(0.09,0.19)$ & $98.89(98.71,99.01)$ & $<0.01$ \\
Hansperger et al., 2016 & $0.13(0.08,0.18)$ & $98.89(98.71,99.00)$ & $<0.01$ \\
\hline
\end{tabular}


Table 3. Cont.

\begin{tabular}{cccc}
\hline Excluded Study & Pooled Prevalence (95\% CI) & $\mathbf{I}^{\mathbf{2}} \mathbf{( 9 5 \%}$ CI) & $p$-Value \\
\hline Oludele et al., 2017 & $0.13(0.08,0.19)$ & $98.89(98.71,99.01)$ & $<0.01$ \\
Simo et al., 2018 & $0.14(0.09,0.20)$ & $98.89(98.74,99.03)$ & $<0.01$ \\
Obonyo et al., 2018 & $0.14(0.09,0.20)$ & $98.89(98.74,99.03)$ & $<0.01$ \\
Tamagda et al., 2018 & $0.13(0.08,0.18)$ & $98.89(98.72,99.01)$ & $<0.01$ \\
Ahmed et al., 2019 & $0.14(0.09,0.19)$ & $98.89(98.74,99.02)$ & $<0.01$ \\
Chipwaza et al., 2020 & $0.14(0.09,0.20)$ & $98.89(98.74,99.03)$ & $<0.01$ \\
Im et al., 2020 & $0.13(0.08,0.18)$ & $98.89(98.57,98.91)$ & $<0.01$ \\
Eldigail et al., 2020 & $0.13(0.09,0.19)$ & $98.89(98.73,99.02)$ & $<0.01$ \\
Shah et al., 2020 & $0.13(0.09,0.18)$ & $98.89(98.39,98.79)$ & $<0.01$ \\
Non-Outbreak Studies $(n=16)$ & & & \\
\hline Hertz et al., 2012 & $0.14(0.10,0.20)$ & $98.89(98.63,98.95)$ & $<0.01$ \\
Vairo et al., 2012 & $0.14(0.09,0.20)$ & $98.89(98.72,99.01)$ & $<0.01$ \\
Chipwaza et al., 2014 & $0.14(0.09,0.20)$ & $98.89(98.74,99.03)$ & $<0.01$ \\
Elduma et al., 2014 & $0.14(0.09,0.20)$ & $98.89(98.74,99.03)$ & $<0.01$ \\
L'Azou et al., 2015 & $0.14(0.09,0.20)$ & $98.89(98.63,98.95)$ & $<0.01$ \\
Konongoi et al., 2016 & $0.14(0.09,0.20)$ & $98.89(98.74,99.03)$ & $<0.01$ \\
Vu et al., 2017 & $0.14(0.09,020)$ & $98.89(98.74,99.03)$ & $<0.01$ \\
Hercik et al., 2018 & $0.14(0.09,0.20)$ & $98.89(98.72,99.02)$ & $<0.01$ \\
Makiala-Mandanda et al., 2018 & $0.14(0.09,0.20)$ & $98.89(98.73,99.02)$ & $<0.01$ \\
Yousseu et al., 2018 & $0.14(0.09,0.20)$ & $98.89(98.74,99.03)$ & $<0.01$ \\
Hamid et al., 2019 & $0.14(0.09,0.20)$ & $98.89(98.74,99.03)$ & $<0.01$ \\
Proesmans et al., 2019 & $0.14(0.09,0.20)$ & $98.89(98.74,99.03)$ & $<0.01$ \\
Ayolabi et al., 2019 & $0.14(0.09,0.20)$ & $98.89(98.74,99.03)$ & $<0.01$ \\
Boyce et al., 2020 & $0.14(0.10,0.20)$ & $98.89(98.46,98.83)$ & $<0.01$ \\
Dieng et al., 2020 & $0.14(0.09,0.20)$ & $98.89(98.74,99.03)$ & $<0.01$ \\
Masika et al., 2020 & $0.14(0.09,020)$ & $98.89(98.70,99.00)$ & $<0.01$ \\
\hline
\end{tabular}

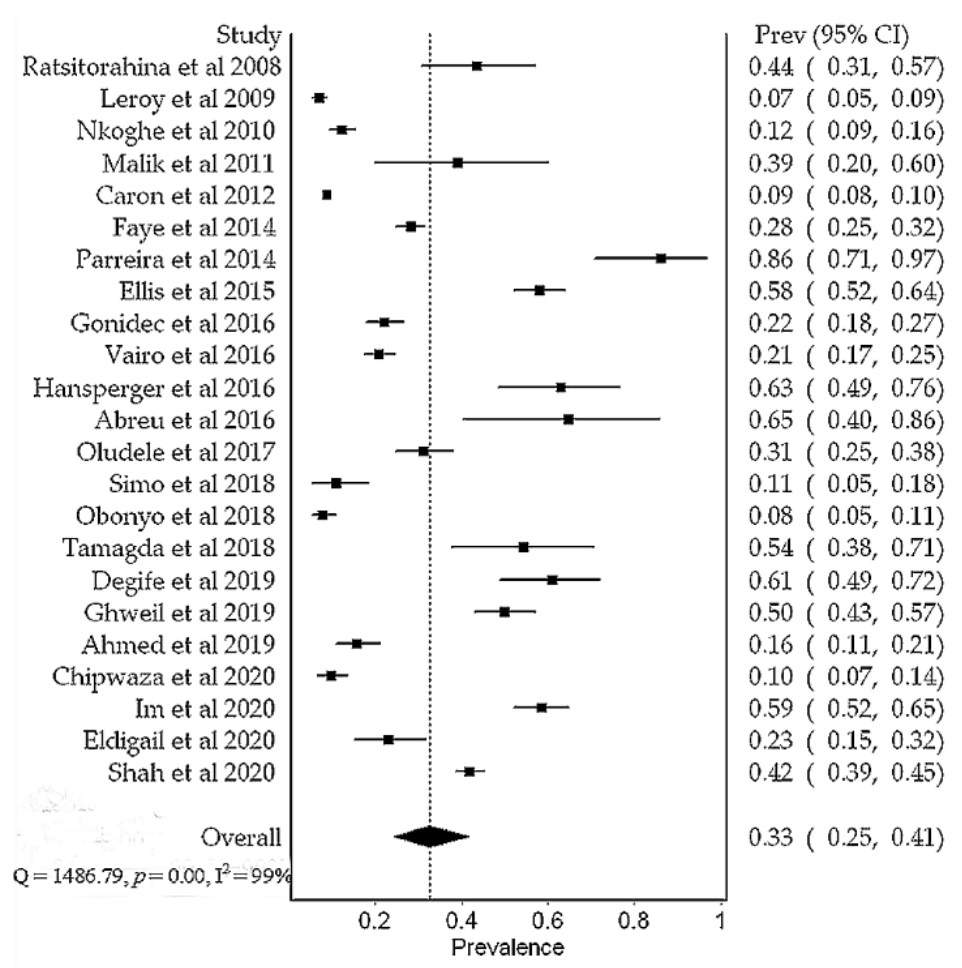

Figure 4. A meta-analysis of dengue virus prevalence in febrile patients during the outbreak periods. 


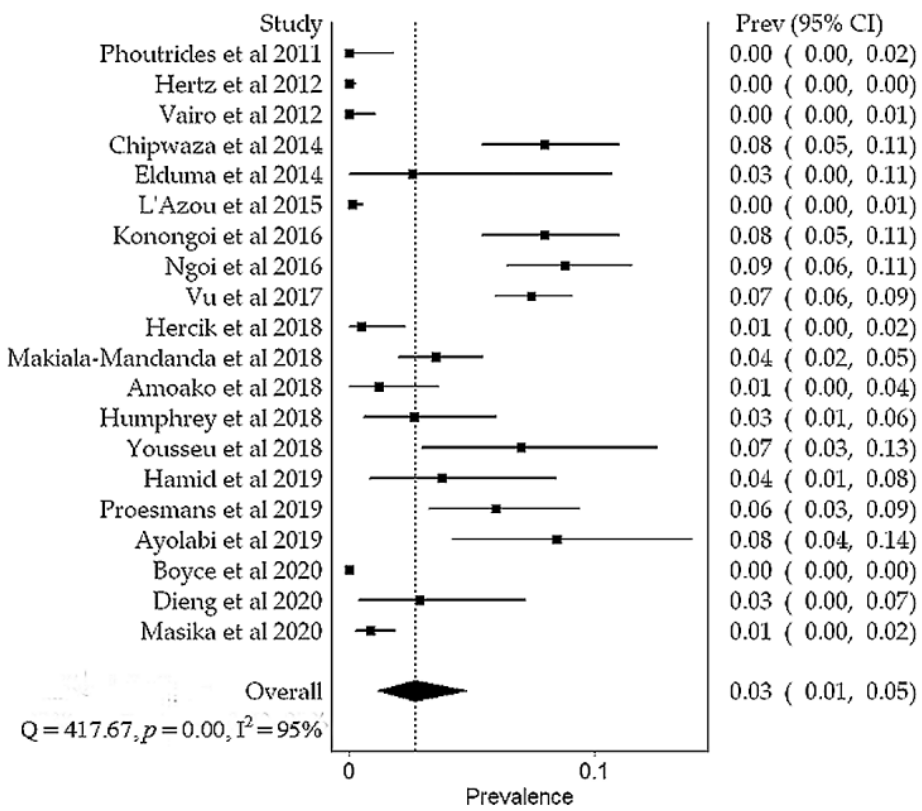

Figure 5. A meta-analysis of dengue virus prevalence in febrile patients during the non-outbreak periods.

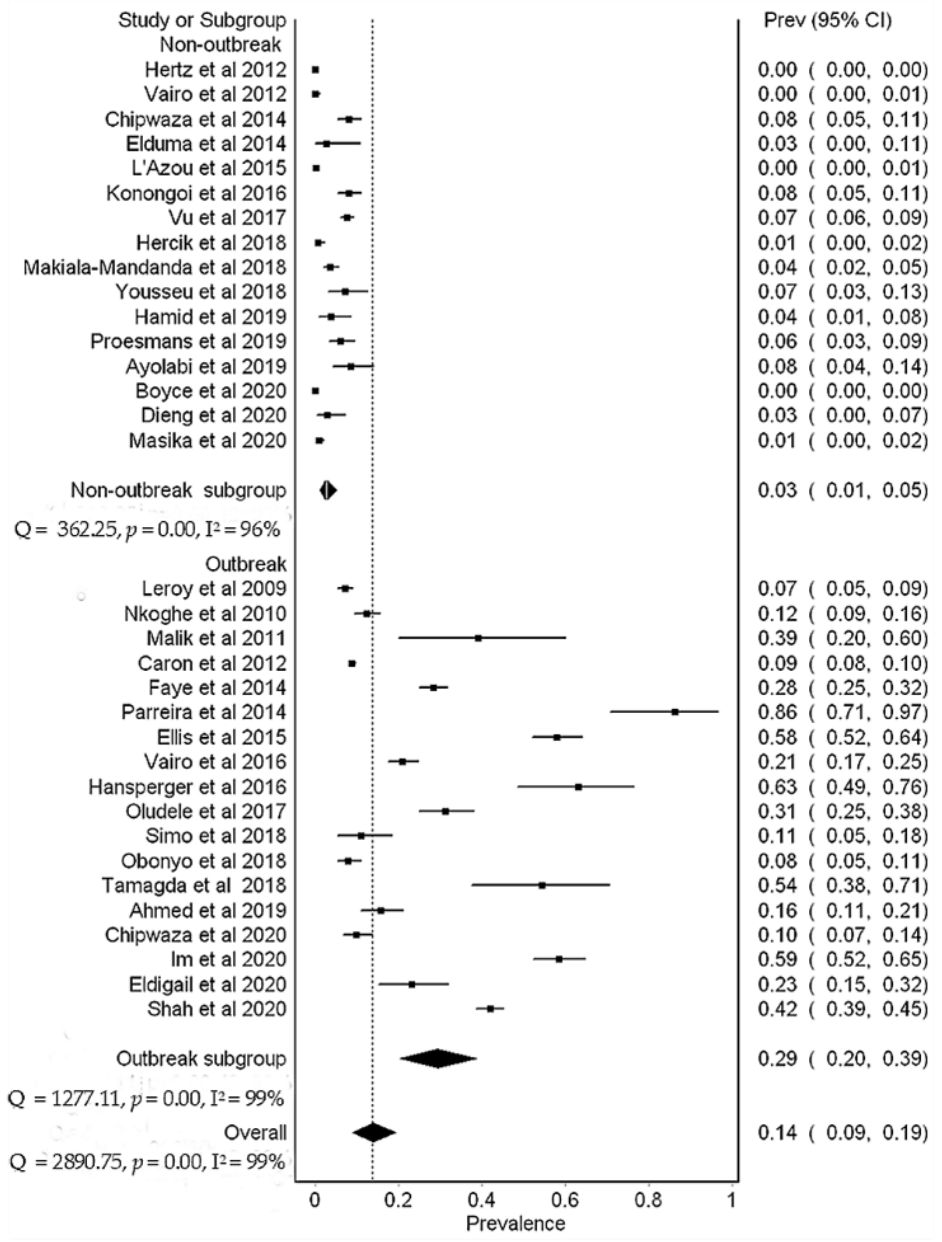

Figure 6. A subgroup meta-analysis of dengue virus prevalence in febrile patients based on prospective cross-sectional studies. 


\subsection{Severe Dengue and Co-Morbidities}

Between 2011 and 2019, a total of 176 severe dengue cases were reported in six countries: Burkina Faso, Côte d'Ivoire, Djibouti, Republic of Sudan, Senegal, and Tanzania. The majority of cases were reported in the Republic of Sudan (126/176) and Burkina Faso $(38 / 176)$. Malaria and dengue co-infections were the most prevalent $(78 \%, 554 / 711)$, followed by dengue and chikungunya co-infections $(16 \%, 114 / 711)$. Other co-morbidities of dengue were yellow fever, measles, pancreatitis, and hepatitis E $(6 \%, 43 / 711)$.

\subsection{Risk Factors}

Evidence from 21 reports published between 2007 and 2020 showed that old age, lack of mosquito control, living in urban areas, climate change, and history of recent travel were the leading risk factors of dengue. Other risk factors were type of occupation, lack of education, low income, and known diabetes mellitus status (Table 4).

Table 4. Potential risk factors of dengue virus infection in Africa ( $n=21$ studies).

\begin{tabular}{ccccc}
\hline Factor & No. Studies & Risk Category & Rank & Reference \\
\hline Increasing old age & 6 & Socio-demographic & 1 & {$[21,48,54,67-69]$} \\
\hline Lack of mosquito control & 6 & Environmental & 1 & {$[27,45,67,70-72]$} \\
\hline Urban residence & 4 & Socio-demographic & 2 & {$[28,54,69,73]$} \\
\hline Climate change & 3 & Ecological & 3 & {$[19,29,74]$} \\
\hline History of recent travel & 3 & Environmental & 3 & {$[27,48,75]$} \\
\hline Occupation type & 2 & Socio-demographic & 4 & {$[68,76]$} \\
\hline Lack of education & 2 & Socio-demographic & 4 & {$[68,77]$} \\
\hline Low income & 1 & Socio-demographic & 5 & {$[54]$} \\
\hline Known diabetes mellitus & 1 & Health & 5 & {$[78]$} \\
\hline
\end{tabular}

\section{Discussion}

This systematic review reports the distribution of outbreaks and the prevalence of dengue in Africa during the outbreak and non-outbreak periods. Our results show that dengue has been reported in 24 of 54 countries and has become endemic, with repeated outbreaks in most of them. Since 1960, all four DENV serotypes (DENV-1-4) caused epidemics in all African sub-regions, with DENV-1 and DENV-2 dominating. Laboratory confirmed outbreaks were reported in 13 African countries, with the East Africa region contributing over $50 \%$ of the epidemics. These observations support evidence previously documented [5,7]. After 2010, severe dengue cases have been increasingly reported in different countries, including Burkina Faso [79], Côte d'Ivoire [80], Djibouti [30], the Republic of Sudan [81], Senegal [22], and Tanzania [31]. The previous report shows that these countries have experienced continuous active DENV transmission in the past decade [5].

Our analysis revealed an increased occurrence of multiple DENV serotypes in Africa during the past decade (2010-2020) (Figure 3), with a greater proportion of serotypes reported in East and West Africa (Figure 2). Concurrent infections with multiple serotypes may pose a risk of severe dengue because lifelong immunity against primary infection by one serotype does not cross-protect subsequent infections by a different serotype. In secondary infection, antibody-dependent enhancement facilitates viral multiplication in the host cells, resulting in severe disease [82]. Expansion of multiple DENV serotypes in Africa may be caused by several factors. International travel of infected people from epidemic and endemic countries has been associated with the introduction of DENV-1 and DENV-3 serotypes in several African countries [83,84]. Spill-over of sylvatic DENV-2 strains from forest Aedes mosquitoes into a human transmission cycle possibly facilitates the spread into urban or new geographical areas with the potential to cause epi- 
demics $[85,86]$. Further, increasing recognition of DENV as the cause of undifferentiated febrile syndromes [87], and the availability of more sensitive and specific molecular-based laboratory tests in the past decade, may have contributed to more detection and reporting of DENV serotypes [51,56,88].

Meta-analysis results show that the overall prevalence of dengue virus in Africa is $14 \%$. This prevalence is relatively higher than the $7 \%$ reported in previous meta-analysis [36]. The discrepancy could be due to differences in a number of prevalence studies included in the meta-analysis. More studies included in this review possibly contributed to an increased number of dengue positive cases. In addition, our review included studies conducted during outbreaks, thus, large studies with a higher proportion of dengue positive cases were expected. During an outbreak, the prevalence of dengue virus was $29 \%$. Our results agree with the $30 \%$ prevalence reported in the previous meta-analysis that included prospective cross-sectional studies conducted during epidemics or following recognized epidemics in the Republic of Sudan [89]. These observations indicate a high burden of dengue of up to $39 \%$ in febrile patients during an outbreak, highlighting the need for routine laboratory dengue diagnosis in tropical Africa.

Low dengue virus prevalence of 3\% in febrile patients was found during the nonoutbreak period (Figure 6). In comparison, our results were relatively lower than values reported in a previous meta-analysis by Simo et al. (2019) involving febrile patients from studies conducted during the non-outbreak period [36]. This difference could be due to the selection and epidemiological contexts of included studies. For instance, inclusion of studies conducted during ongoing epidemics or following epidemics are likely to contribute to a higher number of dengue positive cases [32]. As a result, the overall prevalence could have been overestimated. Despite the low prevalence observed during the non-outbreak period, a burden of up to $5 \%$ in febrile patients is still of a public health concern that needs appropriate interventions. Persistent occurrence of sporadic dengue cases may indicate endemicity, therefore, routine laboratory dengue diagnosis and enhanced mosquito surveillance could help to detect cases early and identify hotspots, respectively. Despite substantial variability $\left(\mathrm{I}^{2}=98.89\right)$ between prospective cross-sectional studies, more than $90 \%$ of the studies had good precision on the overall dengue prevalence (Table 3) and low risk of methodological quality bias (Table S2). The presence of publication bias (Egger's test, $p=0.0022$ ) in the outbreak studies (Figure S1), could be due to small studies with non-significant results not being published.

Co-existing unrecognized co-morbidities can complicate dengue diagnosis and patient management. The findings from previous studies $[90,91]$ show that co-morbidities increase the risk of severe disease and fatal outcomes among dengue patients. Our results show that in the past decade (2010-2020), malaria and dengue co-infections were the most prevalent, followed by dengue and chikungunya co-infection [24,31,40,43,53,56,79,92-95]. A similar occurrence pattern of malaria and dengue co-infection dominance followed by dengue and chikungunya co-infection was previously reported [96]. In Africa, co-morbidities of dengue are not usually diagnosed due to a lack of diagnostic capacity to differentiate dengue from other mosquito-borne acute febrile illnesses such as chikungunya, Zika, and yellow fever. The diseases develop similar non-specific clinical signs and can be co-transmitted with dengue [97]. These findings underscore the need to enhance differential diagnosis of non-malaria febrile illnesses in Africa.

Results from this review show that increasing age, lack of mosquito control, living in an urban area, climate change, and recent history of travel were the leading risk factors of dengue in Africa (Table 4). A high risk of contracting DENV in the old age group may be explained by continuous exposure to Flaviviruses [54,69,72]. The presence and abundance of Aedes mosquito vectors are known to increase risk of dengue exposure [98,99]. Evidence from some studies shows that people living in areas surrounding waste dump sites, opening windows at night, presence of stagnant water at home, households with indoor bathrooms, and living with open water containers were associated with a high risk of dengue [27,45]. Other potential risk factors included occupation type, lack of education, low income, and 
known diabetes mellitus status [48,77]. These findings disclose gaps in individual and environmental practices that could limit Aedes mosquito abundance and spread in African settings.

This review had some limitations. First, we could not establish a meta-analysis of DENV NS1 prevalence due to an inadequate number of studies reporting NS1 prevalence alone. Most studies had overlap data between NS1 and RT-PCR test. Second, a small number of studies $(n<10)$ limited subgroup meta-analysis of dengue prevalence based on setting (community versus healthcare facility), geographical sub-regions, and the design other than prospective cross-sectional. Third, it is possible that some individuals were asymptomatic and could not be detected in the included studies, thus, the number of dengue positive cases may be higher than reported in this review. Despite the limitations, we are confident that our findings provide useful information for clinical practice and public health policy decisions.

\section{Conclusions}

In conclusion, this review reveals a high burden of dengue infection and highlights an increased risk of severe disease in Africa due to the increasing circulation of multiple dengue virus serotypes. We advocate for the need of routine laboratory dengue diagnosis in Africa to facilitate early detection of cases, provision for appropriate patient care, identification of serotypes/genotypes, and outbreak preparedness. It is important to implement effective mosquito surveillance to identify hotspots, and control through the promotion of education on individual behaviours and environmental management practices that can limit the spread of dengue infection in Africa.

Supplementary Materials: The following are available online at https:/ /www.mdpi.com/1999-4 915/13/4/536/s1, Table S1: Quality assessment checklist, Table S2: Risk of bias scores, Figure S1: Funnel plot for the outbreak studies, Figure S2: Funnel plot for the non-outbreak studies.

Author Contributions: Conceptualization, G.O.M. and L.E.G.M.; methodology, G.O.M.; software, G.O.M.; validation, G.O.M., B.N. and C.S.; formal analysis, G.O.M. and G.M.; data curation, G.O.M. and B.N.; writing - original draft preparation, G.O.M. and L.E.G.M.; writing-review and editing, G.O.M., B.N., S.R., C.S., J.J.L., J.T.P., L.E.G.M. and G.M.; visualization, G.O.M., C.S. and G.M.; supervision, L.E.G.M.; J.J.L., J.T.P. and G.M.; funding acquisition, G.M. All authors have read and agreed to the published version of the manuscript.

Funding: This study was funded by the Government of the United Republic of Tanzania through the World Bank (WB-ACE II Grant PAD1436, IDA credit 5799-TZ] to the SACIDS Africa Centre of Excellence for Infectious Diseases at the Sokoine University of Agriculture.

Acknowledgments: We thank the Community of Practice for Emerging and Vector-Borne Disease of the SACIDS Foundation for One Health at Sokoine University of Agriculture for their enthusiasm and discussions throughout the review process.

Conflicts of Interest: The authors declare no conflict of interest. 


\section{Appendix A}

Table A1. Literature search strategy for studies included in the systematic review and meta-analysis. Search date: October to December, 2020.

\section{Key Search Terms:}

(Dengue) [Title] AND (Africa $=$ list of countries) [Title]

(Dengue) [Title] AND (Africa = list of countries) [Title] AND “Outbreak" [Key words]

(Dengue) [Title] AND (Africa = list of countries) [Title] AND "Prevalence" [Key words]

(Dengue) [Title] AND (Africa = list of countries) [Title] AND "Co-morbidies" OR “Co-infections" [Key words]

(Dengue) [Title] AND (Africa = list of countries) [Title] AND “Risk factors" [Key words]

(African countries = "Algeria OR Angola OR Benin OR Botswana OR Burkina Faso OR Burundi OR Cameroon OR Cape Verde OR Central African Republic OR Chad OR Comoros OR Congo (Brazzaville) OR Congo (Democratic Republic) OR Côte d'Ivoire OR Djibouti OR Egypt OR Equatorial Guinea OR Eritrea OR Ethiopia OR Gabon OR Gambia OR Ghana OR Guinea OR Guinea-Bissau OR Kenya OR Lesotho OR Liberia OR Libya OR Madagascar OR Malawi OR Mali OR Mauritania OR Mauritius OR Morocco OR Mozambique OR Namibia OR Niger OR Nigeria OR Reunion OR Rwanda OR Sao Tome and Principe OR Senegal OR Seychelles OR Sierra Leone OR Somalia OR South Africa OR Sudan OR South Sudan OR Swaziland OR Tanzania OR Togo OR Tunisia OR Uganda OR Western Sahara OR Zambia OR Zimbabwe OR Africa OR East Africa OR West Africa OR Southern Africa OR Central Africa OR North Africa"). Publication year limit: 1960-2020 (inclusive).

\section{References}

1. Lambrechts, L.; Scott, T.W.; Gubler, D.J. Consequences of the expanding global distribution of aedes albopictus for dengue virus transmission. PLoS Negl. Trop. Dis. 2010, 4, e646. [CrossRef]

2. Messina, J.P.; Brady, O.J.; Golding, N.; Kraemer, M.U.; Wint, G.W.; Ray, S.E.; Pigott, D.M.; Shearer, F.M.; Johnson, K.; Earl, L. The current and future global distribution and population at risk of dengue. Nat. Microbiol. 2019, 4, 1508-1515. [CrossRef] [PubMed]

3. Bhatt, S.; Gething, P.W.; Brady, O.J.; Messina, J.P.; Farlow, A.W.; Moyes, C.L.; Drake, J.M.; Brownstein, J.S.; Hoen, A.G.; Sankoh, O.; et al. The global distribution and burden of dengue. Nature 2013, 496, 504-507. [CrossRef] [PubMed]

4. Murray, N.E.A.; Quam, M.B.; Wilder-Smith, A. Epidemiology of dengue: Past, present and future prospects. Clin. Epidemiol. 2013, 5, 299-309. [CrossRef]

5. Amarasinghe, A.; Kuritsky, J.N.; Letson, G.W.; Margolis, H.S. Dengue virus infection in Africa. Emerg. Infect. Dis. 2011, 17, 1349-1354. [CrossRef]

6. Guo, C.; Zhou, Z.; Wen, Z.; Liu, Y.; Zeng, C.; Xiao, D.; Ou, M.; Han, Y.; Huang, S.; Liu, D.; et al. Global epidemiology of dengue outbreaks in 1990-2015: A systematic review and Meta-analysis. Front. Cell. Infect. Microbiol. 2017, 7. [CrossRef]

7. Were, F. The dengue situation in Africa. Paediatr. Int. Child Health 2012, 32, 18-21. [CrossRef]

8. LaBeaud, Ad.; Bashir, F.; King, C.H. Measuring the burden of arboviral diseases: The spectrum of morbidity and mortality from four prevalent infections. Popul. Health Metr. 2011, 9, 1-11. [CrossRef] [PubMed]

9. Furuya-Kanamori, L.; Liang, S.; Milinovich, G.; Soares Magalhaes, R.J.; Clements, A.C.A.; Hu, W.; Brasil, P.; Frentiu, F.D.; Dunning, R.; Yakob, L. Co-distribution and co-infection of chikungunya and dengue viruses. BMC Infect. Dis. 2016, 16. [CrossRef]

10. Halsey, E.S.; Baldeviano, G.C.; Edgel, K.A.; Vilcarromero, S.; Sihuincha, M.; Lescano, A.G. Symptoms and immune markers in plasmodium/dengue virus co-infection compared with mono-infection with either in peru. PLoS Negl. Trop. Dis. 2016, 10, e0004646. [CrossRef]

11. Moher, D.; Shamseer, L.; Clarke, M.; Ghersi, D.; Liberati, A.; Petticrew, M.; Shekelle, P.; Stewart, L.A. Preferred reporting items for systematic review and Meta-analysis protocols (PRISMA-P) 2015 statement. Syst. Rev. 2015, 4. [CrossRef]

12. Ouzzani, M.; Hammady, H.; Fedorowicz, Z.; Elmagarmid, A. Rayyan-A web and mobile app for systematic reviews. Syst. Rev. 2016, 5, 210. [CrossRef] [PubMed]

13. Hoy, D.; Brooks, P.; Woolf, A.; Blyth, F.; March, L.; Bain, C.; Baker, P.; Smith, E.; Buchbinder, R. Assessing risk of bias in prevalence studies: Modification of an existing tool and evidence of interrater agreement. J. Clin. Epidemiol. 2012, 65, 934-939. [CrossRef] [PubMed]

14. Ratsitorahina, M.; Harisoa, J.; Ratovonjato, J.; Biacabe, S.; Reynes, J.-M.; Zeller, H.; Raoelina, Y.; Talarmin, A.; Richard, V.; Soares, J.L. Outbreak of dengue and chikungunya fevers, toamasina, madagascar, 2006. Emerg. Infect. Dis. 2008, 14, 1135-1137. [CrossRef] [PubMed]

15. Leroy, E.M.; Nkoghe, D.; Ollomo, B.; Nze-Nkogue, C.; Becquart, P.; Grard, G.; Pourrut, X.; Charrel, R.; Moureau, G.; NdjoyiMbiguino, A.; et al. Concurrent chikungunya and dengue virus infections during simultaneous outbreaks, gabon, 2007. Emerg. Infect. Dis. 2009, 15, 591-593. [CrossRef] [PubMed]

16. Nkoghe, D.; Kassa, R.F.K.; Bisvigou, U.; Caron, M.; Grard, G.; Leroy, E.M. No clinical or biological difference between chikungunya and dengue fever during the 2010 gabonese outbreak. Infect. Dis. Rep. 2012, 4. [CrossRef]

17. Malik, A.; Earhart, K.; Mohareb, E.; Saad, M.; Saeed, M.; Ageep, A.; Soliman, A. Dengue hemorrhagic fever outbreak in children in Port Sudan. J. Infect. Public Health 2011, 4, 1-6. [CrossRef] 
18. Phoutrides, E.K.; Coulibaly, M.B.; George, C.M.; Sacko, A.; Traore, S.; Bessoff, K.; Wiley, M.R.; Kolivras, K.N.; Adelman, Z.; Traore, M.; et al. Dengue virus seroprevalence among febrile patients in Bamako, Mali: Results of a 2006 surveillance study. Vector-Borne Zoonotic Dis. 2011, 11, 1479-1485. [CrossRef]

19. Caron, M.; Paupy, C.; Grard, G.; Becquart, P.; Mombo, I.; Nso, B.B.B.; Kassa Kassa, F.; Nkoghe, D.; Leroy, E.M. Recent introduction and rapid dissemination of chikungunya virus and dengue virus serotype 2 associated with human and mosquito coinfections in Gabon, Central Africa. Clin. Infect. Dis. 2012, 55, e45-e53. [CrossRef]

20. Hertz, J.T.; Munishi, O.M.; Ooi, E.E.; Howe, S.; Lim, W.Y.; Chow, A.; Morrissey, A.B.; Bartlett, J.A.; Onyango, J.J.; Maro, V.P.; et al. Chikungunya and dengue fever among hospitalized febrile patients in Northern Tanzania. Am. J. Trop. Med. Hyg. 2012, 86, 171-177. [CrossRef]

21. Vairo, F.; Nicastri, E.; Meschi, S.; Schepisi, M.S.; Paglia, M.G.; Bevilacqua, N.; Mangi, S.; Sciarrone, M.R.; Chiappini, R.; Mohamed, J. Seroprevalence of dengue infection: A cross-sectional survey in Mainland Tanzania and on Pemba Island, Zanzibar. Int. J. Infect. Dis. 2012, 16, e44-e46. [CrossRef]

22. Faye, O.; Ba, Y.; Faye, O.; Talla, C.; Diallo, D.; Chen, R.; Mondo, M.; Ba, R.; Macondo, E.; Siby, T.; et al. Urban epidemic of dengue virus serotype 3 infection, senegal, 2009. Emerg. Infect. Dis. 2014, 20, 456-459. [CrossRef]

23. Parreira, R.; Conceição, C.; Centeno-Lima, S.; Marques, N.; Saraiva da Cunha, J.; Abreu, C.; Sá, L.; Sarmento, A.; Atouguia, J.; Moneti, V.; et al. Angola's 2013 dengue outbreak: Clinical, laboratory and molecular analyses of cases from four portuguese institutions. J. Infect. Dev. Ctries. 2014, 8, 1210-1215. [CrossRef] [PubMed]

24. Chipwaza, B.; Mugasa, J.P.; Selemani, M.; Amuri, M.; Mosha, F.; Ngatunga, S.D.; Gwakisa, P.S. Dengue and chikungunya fever among viral diseases in outpatient febrile children in Kilosa District Hospital, Tanzania. PLoS Negl. Trop. Dis. 2014, 8. [CrossRef] [PubMed]

25. Elduma, A.H.; Osman, W.M. Dengue and hepatitis E virus infection in pregnant women in eastern sudan, a challenge for diagnosis in an endemic area. Pan Afr. Med. J. 2014, 19. [CrossRef] [PubMed]

26. L'Azou, M.; Succo, T.; Kamagaté, M.; Ouattara, A.; Gilbernair, E.; Adjogoua, E.; Luxemburger, C. Dengue: Etiology of acute febrile illness in Abidjan, Côte d'Ivoire, in 2011-2012. Trans. R. Soc. Trop. Med. Hyg. 2015, 109, 717-722. [CrossRef] [PubMed]

27. Ellis, E.M.; Neatherlin, J.C.; Delorey, M.; Ochieng, M.; Mohamed, A.H.; Mogeni, D.O.; Hunsperger, E.; Patta, S.; Gikunju, S.; Waiboic, L.; et al. A household serosurvey to estimate the magnitude of a dengue outbreak in Mombasa, Kenya, 2013. PLoS Negl. Trop. Dis. 2015, 9. [CrossRef]

28. Konongoi, L.; Ofula, V.; Nyunja, A.; Owaka, S.; Koka, H.; Makio, A.; Koskei, E.; Eyase, F.; Langat, D.; Schoepp, R.J.; et al. Detection of dengue virus serotypes 1, 2 and 3 in selected regions of Kenya: 2011-2014. Virol. J. 2016, 13. [CrossRef]

29. Ngoi, C.N.; Price, M.A.; Fields, B.; Bonventure, J.; Ochieng, C.; Mwashigadi, G.; Hassan, A.S.; Thiong'o, A.N.; Micheni, M.; Mugo, P.; et al. Dengue and chikungunya virus infections among young febrile adults evaluated for acute HIV-1 infection in coastal Kenya. PLoS ONE 2016, 11. [CrossRef] [PubMed]

30. Le Gonidec, E.; Maquart, M.; Duron, S.; Savini, H.; Cazajous, G.; Vidal, P.-O.; Chenilleau, M.-C.; Roseau, J.-B.; Benois, A.; Dehan, C.; et al. Clinical survey of dengue virus circulation in the republic of djibouti between 2011 and 2014 identifies serotype 3 epidemic and recommends clinical diagnosis guidelines for resource limited settings. PLoS Negl. Trop. Dis. 2016, 10. [CrossRef]

31. Vairo, F.; Mboera, L.E.G.; De Nardo, P.; Oriyo, N.M.; Meschi, S.; Rumisha, S.F.; Colavita, F.; Mhina, A.; Carletti, F.; Mwakapeje, E.; et al. Clinical, virologic, and epidemiologic characteristics of dengue outbreak, Dar Es Salaam, Tanzania, 2014. Emerg. Infect. Dis. 2016, 22, 895-899. [CrossRef]

32. Hunsperger, E.A.; Sharp, T.M.; Lalita, P.; Tikomaidraubuta, K.; Cardoso, Y.R.; Naivalu, T.; Khan, A.S.; Marfel, M.; Hancock, W.T.; Tomashek, K.M.; et al. Use of a rapid test for diagnosis of dengue during suspected dengue outbreaks in resource-limited regions. J. Clin. Microbiol. 2016, 54, 2090-2095. [CrossRef] [PubMed]

33. Abreu, C.; Silva-Pinto, A.; Lazzara, D.; Sobrinho-Simões, J.; Guimarães, J.T.; Sarmento, A. Imported dengue from 2013 angola outbreak: Not just serotype 1 was detected. J. Clin. Virol. 2016, 79, 77-79. [CrossRef]

34. Vu, D.M.; Mutai, N.; Heath, C.J.; Vulule, J.M.; Mutuku, F.M.; Ndenga, B.A.; LaBeaud, A.D. Unrecognized dengue virus infections in children, western Kenya, 2014-2015. Emerg. Infect. Dis. 2017, 23, 1915-1917. [CrossRef] [PubMed]

35. Oludele, J.; Lesko, B.; Gundane, I.M.; de Bruycker-Nogueira, F.; Ali, S.; Mula, F.; Chelene, I.; Falk, K.I.; Dos Santos, F.B.; Gudo, E.S. Dengue virus serotype 2 established in northern Mozambique (2015-2016). Am. J. Trop. Med. Hyg. 2017, 97, 1418-1422. [CrossRef]

36. Simo, F.B.N.; Bigna, J.J.; Kenmoe, S.; Ndangang, M.S.; Temfack, E.; Moundipa, P.F.; Demanou, M. Dengue virus infection in people residing in Africa: A systematic review and meta-analysis of prevalence studies. Sci. Rep. 2019, 9, 1-9. [CrossRef] [PubMed]

37. Hercik, C.; Cosmas, L.; Mogeni, O.D.; Wamola, N.; Kohi, W.; Houpt, E.; Liu, J.; Ochieng, C.; Onyango, C.; Fields, B. A combined syndromic approach to examine viral, bacterial, and parasitic agents among febrile patients: A pilot study in Kilombero, Tanzania. Am. J. Trop. Med. Hyg. 2018, 98, 625-632. [CrossRef]

38. Obonyo, M.; Fidhow, A.; Ofula, V. Investigation of laboratory confirmed dengue outbreak in north-eastern Kenya, 2011. PLoS ONE 2018, 13, e0198556. [CrossRef]

39. Makiala-Mandanda, S.; Ahuka-Mundeke, S.; Abbate, J.L.; Pukuta-Simbu, E.; Nsio-Mbeta, J.; Berthet, N.; Leroy, E.M.; Becquart, P.; Muyembe-Tamfum, J.-J. Identification of dengue and chikungunya cases among suspected cases of yellow fever in the Democratic Republic of the Congo. Vector-Borne Zoonotic Dis. 2018, 18, 364-370. [CrossRef] 
40. Amoako, N.; Duodu, S.; Dennis, F.E.; Bonney, J.H.K.; Asante, K.P.; Ameh, J.; Mosi, L.; Hayashi, T.; Agbosu, E.E.; Pratt, D.; et al. Detection of dengue virus among children with suspected Malaria, Accra, Ghana. Emerg. Infect. Dis. 2018, 24, $1544-1547$. [CrossRef]

41. Bonney, J.H.K.; Hayashi, T.; Dadzie, S.; Agbosu, E.; Pratt, D.; Nyarko, S.; Asiedu-Bekoe, F.; Ido, E.; Sarkodie, B.; Ohta, N.; et al. Molecular detection of dengue virus in patients suspected of ebola virus disease in Ghana. PLoS ONE 2018, 13. [CrossRef] [PubMed]

42. Tarnagda, Z.; Cissé, A.; Bicaba, B.W.; Diagbouga, S.; Sagna, T.; Ilboudo, A.K.; Tialla, D.; Lingani, M.; Sondo, K.A.; Yougbaré, I.; et al. Dengue fever in Burkina Faso, 2016. Emerg. Infect. Dis. 2018, 24, 170-172. [CrossRef]

43. Yousseu, F.B.S.; Nemg, F.B.S.; Ngouanet, S.A.; Mekanda, F.M.O.; Demanou, M. Detection and serotyping of dengue viruses in febrile patients consulting at the new-bell district hospital in Douala, Cameroon. PLoS ONE 2018, 13, e0204143. [CrossRef]

44. Hamid, Z.; Hamid, T.; Alsedig, K.; Abdallah, T.; Elaagip, A.; Ahmed, A.; Khalid, F.; Hamid, M.A. Molecular investigation of dengue virus serotype 2 circulation in Kassala State, Sudan. Jpn. J. Infect. Dis. 2019, 72, 58-61. [CrossRef]

45. Degife, L.H.; Worku, Y.; Belay, D.; Bekele, A.; Hailemariam, Z. Factors associated with dengue fever outbreak in dire dawa administration City, October, 2015, Ethiopia-Case control study. BMC Public Health 2019, 19, 650. [CrossRef]

46. Ghweil, A.A.; Osman, H.A.; Khodeary, A.; Okasha, A.; Hassan, M.H. Relative frequency of acute pancreatitis from dengue outbreaks as a late complication, in Egypt. VirusDis 2019, 30, 498-503. [CrossRef]

47. Ahmed, A.; Elduma, A.; Magboul, B.; Higazi, T.; Ali, Y. The first outbreak of dengue fever in greater darfur, western Sudan. Trop. Med. Infect. Dis. 2019, 4, 43. [CrossRef] [PubMed]

48. Proesmans, S.; Katshongo, F.; Milambu, J.; Fungula, B.; Muhindo Mavoko, H.; Ahuka-Mundeke, S.; Inocêncio da Luz, R.; Van Esbroeck, M.; Ariën, K.K.; Cnops, L.; et al. Dengue and chikungunya among outpatients with acute undifferentiated fever in Kinshasa, Democratic Republic of Congo: A cross-sectional study. PLoS Negl. Trop. Dis. 2019, 13. [CrossRef]

49. Ayolabi, C.I.; Olusola, B.A.; Ibemgbo, S.A.; Okonkwo, G.O. Detection of dengue viruses among febrile patients in Lagos, Nigeria and phylogenetics of circulating dengue serotypes in Africa. Infect. Genet. Evol. 2019, 75. [CrossRef]

50. Boyce, R.M.; Collins, M.; Muhindo, R.; Nakakande, R.; Ciccone, E.J.; Grounds, S.; Espinoza, D.; Zhu, Y.; Matte, M.; Ntaro, M.; et al. Dengue in western Uganda: A prospective cohort of children presenting with undifferentiated febrile illness. BMC Infect. Dis. 2020, 20. [CrossRef] [PubMed]

51. Chipwaza, B.; Sumaye, R.D.; Weisser, M.; Gingo, W.; Yeo, N.K.-W.; Amrun, S.N.; Okumu, F.O.; Ng, L.F.P. Occurrence of 4 dengue virus serotypes and chikungunya virus in Kilombero Valley, Tanzania, during the dengue outbreak in 2018. Open Forum Infect. Dis. 2021, 8. [CrossRef]

52. Dieng, I.; Hedible, B.G.; Diagne, M.M.; El Wahed, A.A.; Diagne, C.T.; Fall, C.; Richard, V.; Vray, M.; Weidmann, M.; Faye, O. Mobile laboratory reveals the circulation of dengue virus serotype I of Asian Origin in medina gounass (guediawaye), senegal. Diagnostics 2020, 10, 408. [CrossRef]

53. Im, J.; Balasubramanian, R.; Ouedraogo, M.; Wandji Nana, L.R.; Mogeni, O.D.; Jeon, H.J.; van Pomeren, T.; Haselbeck, A.; Lim, J.K.; Prifti, K.; et al. The epidemiology of dengue outbreaks in 2016 and 2017 in Ouagadougou, Burkina Faso. Heliyon 2020, 6. [CrossRef]

54. Eldigail, M.H.; Abubaker, H.A.; Khalid, F.A.; Abdallah, T.M.; Adam, I.A.; Adam, G.K.; Babiker, R.A.; Ahmed, M.E.; Haroun, E.M.; Aradaib, I.E. Recent transmission of dengue virus and associated risk facors among residents of Kassala State, Eastern Sudan BMC Public Health 2020, 20. [CrossRef] [PubMed]

55. Masika, M.M.; Korhonen, E.M.; Smura, T.; Uusitalo, R.; Vapalahti, K.; Mwaengo, D.; Jääskeläinen, A.J.; Anzala, O.; Vapalahti, O.; Huhtamo, E. Detection of dengue virus type 2 of indian origin in acute febrile patients in Rural Kenya. PLoS Negl. Trop. Dis. 2020, 14. [CrossRef] [PubMed]

56. Shah, M.M.; Ndenga, B.A.; Mutuku, F.M.; Vu, D.M.; Grossi-Soyster, E.N.; Okuta, V.; Ronga, C.O.; Chebii, P.K.; Maina, P.; Jembe, Z.; et al. High dengue burden and circulation of 4 virus serotypes among children with undifferentiated fever, Kenya, 2014-2017. Emerg. Infect. Dis. 2020, 26, 2638. [CrossRef] [PubMed]

57. Carey, D. Dengue viruses from febrile patients in Nigeria, 1964-68. The Lancet 1971, 297, 105-106. [CrossRef]

58. Metselaar, D.; Grainger, C.R.; Oei, K.G.; Reynolds, D.G.; Pudney, M.; Leake, C.J.; Tukei, P.M.; D'Offay, R.M.; Simpson, D.I.H. An outbreak of type 2 dengue fever in the seychelles, probably transmitted by aedes albopictus (skuse). Bull. World Health Organ. 1980, 58, 937-943.

59. Saluzzo, J.F.; Cornet, M.; Castagnet, P.; Rey, C.; Digoutte, J.P. Isolation of dengue 2 and dengue 4 viruses from patients in senegal. Trans. R. Soc. Trop. Med. Hyg. 1986, 80, 5. [CrossRef]

60. Botros, B.A.M.; Watts, D.M.; Soliman, A.K.; Salib, A.W.; Moussa, M.I.; Mursal, H.; Douglas, C.; Farah, M. Serological evidence of dengue fever among Refugees, Hargeysa, Somalia. J. Med. Virol. 1989, 29, 79-81. [CrossRef]

61. Home-ProMED. Available online: https:/ / promedmail.org/ (accessed on 3 December 2020).

62. WHO. Disease Outbreak News (DONs). Available online: http://www.who.int/csr/don/en/ (accessed on 3 December 2020).

63. Issack, M.I.; Pursem, V.N.; Barkham, T.M.S.; Ng, L.-C.; Inoue, M.; Manraj, S.S. Reemergence of dengue in mauritius. Emerg. Infect. Dis. 2010, 16, 716-718. [CrossRef]

64. Seidahmed, O.M.E.; Siam, H.A.M.; Soghaier, M.A.; Abubakr, M.; Osman, H.A.; Abd Elrhman, L.S.; Elmagbol, B.; Velayudhan, R. Dengue vector control and surveillance during a major outbreak in a coastal red sea area in Sudan. East. Mediterr. Health J. 2012, 18, 1217-1224. [CrossRef] [PubMed] 
65. Woyessa, A.B.; Megensha, M.; Kassa, W.; Kifle, E.; Wondabeku, M.; Girmay, A.; Kebede, A.; Jima, D. The first acute febrile illness investigation associated with dengue fever in Ethiopia, 2013: A descriptive analysis. Ethiop. J. Health Dev. 2020, 28, 155-161.

66. Vincent, M.; Larrieu, S.; Vilain, P.; Etienne, A.; Solet, J.-L.; François, C.; Roquebert, B.; Jaffar Bandjee, M.-C.; Filleul, L.; Menudier, L. From the threat to the large outbreak: Dengue on reunion Island, 2015 to 2018. Euro Surv 2019, 24. [CrossRef] [PubMed]

67. Demanou, M.; Pouillot, R.; Grandadam, M.; Boisier, P.; Kamgang, B.; Hervé, J.P.; Rogier, C.; Rousset, D.; Paupy, C. Evidence of dengue virus transmission and factors associated with the presence of anti-dengue virus antibodies in humans in three major towns in cameroon. PLoS Negl. Trop. Dis. 2014, 8. [CrossRef]

68. Nasir, I.A.; Agbede, O.O.; Dangana, A.; Baba, M.; Haruna, A.S. Dengue Virus Non-Structural Protein-1 Expression and Associated Risk Factors among Febrile Patients Attending University of Abuja Teaching Hospital, Nigeria. Virus Res. 2016, 230, 7-12. [CrossRef]

69. Sawadogo, S.; Baguiya, A.; Yougbare, F.; Bicaba, B.W.; Nebie, K.; Millogo, T.; Kamba, I.; Kaba, L.; Sangare, L.; Kafando, E.; et al. Seroprevalence and factors associated with IgG Anti-DENV positivity in blood donors in burkina faso during the 2016 dengue outbreak and implications for blood supply. Transfus. Med. 2020, 30, 37-45. [CrossRef]

70. Ferede, G.; Tiruneh, M.; Abate, E.; Wondimeneh, Y.; Damtie, D.; Gadisa, E.; Howe, R.; Aseffa, A.; Tessema, B. A serologic study of dengue in northwest Ethiopia: Suggesting preventive and control measures. PLoS Negl. Trop. Dis. 2018, 12, e0006430. [CrossRef]

71. Omatola, C.A.; Onoja, A.B.; Moses, E.; Mahmud, M.; Mofolorunsho, C.K. Dengue in parts of the guinea savannah region of nigeria and the risk of increased transmission. Int. Health 2020, 1-5, Online Ahead of Print. [CrossRef]

72. Elaagip, A.; Alsedig, K.; Altahir, O.; Ageep, T.; Ahmed, A.; Siam, H.A.; Samy, A.M.; Mohamed, W.; Khalid, F.; Gumaa, S.; et al. Seroprevalence and associated risk factors of dengue fever in Kassala State, Eastern Sudan. PLoS Negl. Trop. Dis. 2020, 14, e0008918. [CrossRef]

73. Vairo, F.; Nicastri, E.; Yussuf, S.M.; Cannas, A.; Meschi, S.; Mahmoud, M.A.; Mohamed, A.H.; Maiko, P.M.; De Nardo, P.; Bevilacqua, N.; et al. IgG against dengue virus in healthy blood donors, Zanzibar, Tanzania. Emerg. Infect. Dis. 2014, 20, 465-468. [CrossRef]

74. Mweya, C.N.; Kimera, S.I.; Stanley, G.; Misinzo, G.; Mboera, L.E.G. Climate change influences potential distribution of infected aedes aegypti co-occurrence with dengue epidemics risk areas in Tanzania. PLoS ONE 2016, 11. [CrossRef] [PubMed]

75. Mazaba-Liwewe, M.L.; Siziya, S.; Monze, M.; Mweene-Ndumba, I.; Masaninga, F.; Songolo, P.; Malama, C.; Chizema, E.; Mwaba, P.; Babaniyi, O.A. First sero-prevalence of dengue fever specific immunoglobulin $\mathrm{G}$ antibodies in western and north-western provinces of Zambia: A population based cross sectional study. Virol. J. 2014, 11, 135. [CrossRef]

76. Mustapha, J.O.; Emeribe, A.U.; Nasir, I.A. Survey of malaria and anti-dengue virus IgG among febrile HIV-infected patients attending a tertiary hospital in Abuja, Nigeria. HIVAIDS Auckl. NZ 2017, 9, 145-151. [CrossRef]

77. Ochieng, C.; Ahenda, P.; Vittor, A.Y.; Nyoka, R.; Gikunju, S.; Wachira, C.; Waiboci, L.; Umuro, M.; Kim, A.A.; Nderitu, L.; et al. Seroprevalence of infections with dengue, rift valley fever and chikungunya viruses in Kenya, 2007. PLoS ONE 2015, 10. [CrossRef] [PubMed]

78. Boillat-Blanco, N.; Klaassen, B.; Mbarack, Z.; Samaka, J.; Mlaganile, T.; Masimba, J.; Franco Narvaez, L.; Mamin, A.; Genton, B.; Kaiser, L.; et al. Dengue fever in Dar Es Salaam, Tanzania: Clinical features and outcome in populations of black and non-black racial category. BMC Infect. Dis. 2018, 18. [CrossRef] [PubMed]

79. Sondo, K.A.; Ouattara, A.; Diendéré, E.A.; Diallo, I.; Zoungrana, J.; Zémané, G.; Da, L.; Gnamou, A.; Meda, B.; Poda, A.; et al. Dengue infection during pregnancy in Burkina Faso: A cross-sectional study. BMC Infect. Dis. 2019, 19. [CrossRef] [PubMed]

80. Aoussi, E.B.F.; Ehui, E.; Kassi, N.A.; Kouakou, G.; Nouhou, Y.; Adjogoua, E.V.; Eholié, S.; Bissagnéné, E. Seven native cases of dengue in Abidjan, Ivory Coast. Médecine Mal. Infect. 2014, 44, 433-436. [CrossRef]

81. Pulsan, F.; Sobi, K.; Anga, G.; Vince, J.; Duke, T. An outbreak of dengue fever in children in the national capital district of papua new guinea in 2016. Paediatr. Int. Child Health 2020, 40, 177-180. [CrossRef]

82. Katzelnick, L.C.; Gresh, L.; Halloran, M.E.; Mercado, J.C.; Kuan, G.; Gordon, A.; Balmaseda, A.; Harris, E. Antibody-dependent enhancement of severe dengue disease in humans. Science 2017, 358, 929-932. [CrossRef]

83. Dieng, I.; Cunha, M. dos P.; Diagne, M.M.; Sembène, P.M.; Zanotto, P.M. de A.; Faye, O.; Faye, O.; Sall, A.A. Origin and spread of the dengue virus type 1, genotype $\mathrm{V}$ in senegal, 2015-2019. Viruses 2021, 13, 57. [CrossRef] [PubMed]

84. Messer, W.B.; Gubler, D.J.; Harris, E.; Sivananthan, K.; de Silva, A.M. Emergence and global spread of a dengue serotype 3, subtype III virus. Emerg. Infect. Dis. 2003, 9, 800-809. [CrossRef]

85. Franco, L.; Palacios, G.; Martinez, J.A.; Sanchez-Seco, M.P.; Martın, D.; Lipkin, W.I.; Tenorio, A. First report of sylvatic DENV-2associated dengue hemorrhagic fever in West Africa. PLoS Negl. Trop Dis. 2011, 5, 7. [CrossRef]

86. Vasilakis, N.; Tesh, R.B.; Weaver, S.C. Sylvatic dengue virus type 2 activity in humans, Nigeria, 1966. Emerg. Infect. Dis. 2008, 14, 502-504. [CrossRef] [PubMed]

87. Maze, M.J.; Bassat, Q.; Feasey, N.A.; Mandomando, I.; Musicha, P.; Crump, J.A. The epidemiology of febrile illness in sub-saharan Africa: Implications for diagnosis and management. Clin. Microbiol. Infect. 2018, 24, 808-814. [CrossRef] [PubMed]

88. Jaenisch, T.; Junghanss, T.; Wills, B.; Brady, O.J.; Eckerle, I.; Farlow, A.; Hay, S.I.; McCall, P.J.; Messina, J.P.; Ofula, V.; et al. Dengue expansion in Africa-Not recognized or not happening? Emerg. Infect. Dis. 2014, 20. [CrossRef] [PubMed]

89. Elduma, A.H.; LaBeaud, A.D.; A. Plante, J.; Plante, K.S.; Ahmed, A. High seroprevalence of dengue virus infection in Sudan: Systematic review and meta-analysis. Trop. Med. Infect. Dis. 2020, 5, 120. [CrossRef] 
90. Werneck, G.L.; Macias, A.E.; Mascarenas, C.; Coudeville, L.; Morley, D.; Recamier, V.; Guergova-Kuras, M.; Puentes-Rosas, E.; Baurin, N.; Toh, M.-L. Comorbidities increase in-hospital mortality in dengue patients in Brazil. Mem. Inst. Oswaldo Cruz 2018, 113. [CrossRef]

91. Saqib, M.A.N.; Rafique, I.; Bashir, S.; Salam, A.A. A retrospective analysis of dengue fever case management and frequency of co-morbidities associated with deaths. BMC Res. Notes 2014, 7, 205. [CrossRef]

92. Oyero, O.G.; Ayukekbong, J.A. High dengue NS1 antigenemia in febrile patients in Ibadan, Nigeria. Virus Res. 2014, 191, 59-61. [CrossRef] [PubMed]

93. Dariano, D.F.; Taitt, C.R.; Jacobsen, K.H.; Bangura, U.; Bockarie, A.S.; Bockarie, M.J.; Lahai, J.; Lamin, J.M.; Leski, T.A.; Yasuda, C.; et al. Surveillance of vector-borne infections (Chikungunya, Dengue, and Malaria) in Bo, Sierra Leone, 2012-2013. Am. J. Trop. Med. Hyg. 2017, 97, 1151-1154. [CrossRef] [PubMed]

94. Kolawole, O.M.; Seriki, A.A.; Irekeola, A.A.; Bello, K.E.; Adeyemi, O.O. Dengue virus and malaria concurrent infection among febrile subjects within Ilorin Metropolis, Nigeria. J. Med. Virol. 2017, 89, 1347-1353. [CrossRef]

95. Onyedibe, K. A cross sectional study of dengue virus infection in febrile patients presumptively diagnosed of malaria in Maiduguri and Jos Plateau, Nigeria. Malawi Med. J. 2018, 30, 276. [CrossRef]

96. Salam, N.; Mustafa, S.; Hafiz, A.; Chaudhary, A.A.; Deeba, F.; Parveen, S. Global prevalence and distribution of coinfection of malaria, dengue and chikungunya: A systematic review. BMC Public Health 2018, 18, 710. [CrossRef] [PubMed]

97. Petti, C.A.; Polage, C.R.; Quinn, T.C.; Ronald, A.R.; Sande, M.A. Laboratory medicine in Africa: A barrier to effective health care. Clin. Infect. Dis. 2006, 42, 377-382. [CrossRef] [PubMed]

98. Msellemu, D.; Gavana, T.; Ngonyani, H.; Mlacha, Y.P.; Chaki, P.; Moore, S.J. Knowledge, attitudes and bite prevention practices and estimation of productivity of vector breeding sites using a Habitat Suitability Score (HSS) among households with confirmed dengue in the 2014 outbreak in Dar Es Salaam, Tanzania. PLoS Negl. Trop. Dis. 2020, 14, e0007278. [CrossRef]

99. Higa, Y.; Futami, K.; Lázaro, M.A.F.; Minakawa, N.; Gudo, E.S. Abundant aedes (stegomyia) aegypti aegypti mosquitoes in the 2014 dengue outbreak area of Mozambique. Trop. Med. Health 2015, 43, 107-109. [CrossRef] 